\title{
PRESTABLE IDEALS AND SAGBI BASES
}

\author{
HIDEFUMI OHSUGI and TAKAYUKI HIBI
}

\begin{abstract}
In order to find a reasonable class of squarefree monomial ideals $I$ for which the toric ideal of the Rees algebra of $I$ has a quadratic Gröbner basis, the concept of prestable ideals will be introduced. Prestable ideals arising from finite pure posets together with their application to Sagbi bases will be discussed.
\end{abstract}

\section{Introduction}

Let $R=K\left[x_{1}, \ldots, x_{n}\right]$ denote the polynomial ring in $n$ variables over a field $K$ with each $\operatorname{deg} x_{i}=1$ and let $I \subset R$ be an ideal which is generated by monomials $u_{1}, \ldots, u_{m}$ with $\operatorname{deg} u_{1}=\cdots=\operatorname{deg} u_{m}$. The Rees algebra of $I$ is the subalgebra $\mathscr{R}(I)=K\left[x_{1}, \ldots, x_{n}, u_{1} t, \ldots, u_{m} t\right]$ of $R[t]$. Let $A=$ $K\left[x_{1}, \ldots, x_{n}, y_{1}, \ldots, y_{m}\right]=R\left[y_{1}, \ldots, y_{m}\right]$ denote the polynomial ring over $K$ and define the surjective homomorphism $\pi: A \rightarrow \mathscr{R}(I)$ by setting $\pi\left(x_{i}\right)=$ $x_{i}$ and $\pi\left(y_{j}\right)=u_{j} t$. The toric ideal $J_{\mathscr{R}(I)}$ of $\mathscr{R}(I)$ is the kernel of $\pi$. Blum [1] proved that if $\mathscr{R}(I)$ is Koszul, then all powers of $I$ have linear resolutions. Thus in particular if $J_{\mathscr{R}(I)}$ has a quadratic Gröbner basis, then all powers of $I$ have linear resolutions. However, the existence of a quadratic Gröbner basis of $J_{\mathscr{R}(I)}$ is a rather strong condition which guarantees that all powers of $I$ have linear resolutions. In [8] a much weaker condition, called the $x$-condition, for $J_{\mathscr{R}(I)}$ is introduced and it is proved that if $J_{\mathscr{R}(I)}$ satisfies the $x$-condition, then all powers of $I$ have linear resolutions.

In the present paper, a new class of monomial ideals, the class of prestable ideals, which contains the stable ideals [4] is introduced. If $I$ is prestable, then $J_{\mathscr{R}(I)}$ satisfies the $x$-condition and all powers of $I$ have linear resolutions. See Corollary 1.5. We then discuss a class of prestable squarefree monomial ideals $I$ arising from finite pure posets (partially ordered sets) such that $J_{\mathscr{R}(I)}$ has a quadratic Gröbner basis. See Theorem 2.1 and Corollary 2.2. Finally, as one of the applications of such prestable ideals coming from finite pure posets, Sagbi bases of the algebras studied in [5] will be determined. See Theorem 3.2.

Received August 25, 2003. 


\section{Prestable sets}

Let $R=K\left[x_{1}, \ldots, x_{n}\right]$ denote the polynomial ring in $n$ variables over a field $K$ with each $\operatorname{deg} x_{i}=1$, and write $V_{n}^{(d)}$ for the set of all monomials of $R$ of degree $d$.

A nonempty subset $\mathcal{N}=\left\{u_{1}, u_{2}, \ldots, u_{m}\right\} \subset V_{n}^{(d)}$ is said to be a prestable set if $\mathcal{N}$ possesses the exchange property (*) as follows:

(*) For all $N=1,2, \ldots$ and for any two monomials $\prod_{j=1}^{N} u_{i_{j}}$ and $\prod_{\ell=1}^{N} u_{k_{\ell}}$ with $\prod_{j=1}^{N} u_{i_{j}}=x_{1}^{a_{1}} x_{2}^{a_{2}} \cdots x_{n}^{a_{n}}$ and $\prod_{\ell=1}^{N} u_{k_{\ell}}=x_{1}^{b_{1}} x_{2}^{b_{2}} \cdots x_{n}^{b_{n}}$ such that $a_{1}=b_{1}, \ldots, a_{q-1}=b_{q-1}$ and $a_{q}<b_{q}$ for some $1 \leq q<n$, there exist $1 \leq j \leq N$ and $q<p \leq n$ such that $x_{q}\left(u_{i_{j}} / x_{p}\right) \in \mathcal{N}$.

One of the most fundamental classes of prestable sets is

EXAMPLE 1.1. Recall that a set of monomials $\mathcal{N} \subset V_{n}^{(d)}$ is stable if, for all $u \in \mathscr{N}$ and for all $i<m(u)$, one has $x_{i}\left(u / x_{m(u)}\right) \in \mathcal{N}$. Here $m(u)$ is the largest $i$ for which $x_{i}$ divides $u$. A stable set $\mathcal{N}$ is prestable. In fact, if $\prod_{j=1}^{N} u_{i_{j}}=x_{1}^{a_{1}} x_{2}^{a_{2}} \cdots x_{n}^{a_{n}}$ and $\prod_{\ell=1}^{N} u_{k_{\ell}}=x_{1}^{b_{1}} x_{2}^{b_{2}} \cdots x_{n}^{b_{n}}$, with each $u_{i_{j}}, u_{k_{\ell}} \in$ $\mathcal{N}$ such that $a_{1}=b_{1}, \ldots, a_{q-1}=b_{q-1}$ and $a_{q}<b_{q}$, then there is $p>q$ with $a_{p}>b_{p}$. In particular $x_{p}$ divides some $u_{i_{j}}$. Since $m\left(u_{i_{j}}\right) \geq p>q$ and since $\mathcal{N}$ is stable, one has $x_{q}\left(u_{i_{j}} / x_{m\left(u_{i_{j}}\right)}\right) \in \mathscr{N}$. We will give a prestable set which is not stable. See Example 2.3.

Let $I \subset R$ be an ideal generated by monomials of degree $d$ and $G(I)$ the minimal system of monomial generators of $I$, i.e., $G(I)=I \cap V_{n}^{(d)}$. We say that $I$ is prestable if $G(I) \subset V_{n}^{(d)}$ is a prestable set. The Rees algebra of $I$ is the semigroup ring

$$
\mathscr{R}(I)=K\left[x_{1}, \ldots, x_{n},\{u t\}_{u \in G(I)}\right] \quad\left(\subset R[t]=K\left[x_{1}, \ldots, x_{n}, t\right]\right) .
$$

Let $A=K\left[x_{1}, \ldots, x_{n},\left\{y_{u}\right\}_{u \in G(I)}\right]=R\left[\left\{y_{u}\right\}_{u \in G(I)}\right]$ denote the polynomial ring over $K$ with each $\operatorname{deg} x_{i}=\operatorname{deg} y_{u}=1$. The toric ideal of $\mathscr{R}(I)$ is the ideal $J_{\mathscr{R}(I)} \subset A$ which is the kernel of the surjective homomorphism $\pi: A \rightarrow \mathscr{R}(I)$ defined by setting $\pi\left(x_{i}\right)=x_{i}$ for each $1 \leq i \leq n$ and $\pi\left(y_{u}\right)=u t$ for each $u \in G(I)$.

Let $<^{(\sharp)}$ be an arbitrary monomial order on the polynomial ring $K\left[\left\{y_{u}\right\}_{u \in G(I)}\right]$ and $<_{l e x}$ the lexicographic order on $R$ induced by $x_{1}>x_{2}>\cdots>x_{n}$. We then introduce the new monomial order $<_{l e x}^{(\sharp)}$ on $A$ by setting $\mathbf{x}^{\mathbf{a}} \mathbf{y}^{\mathbf{b}}<_{l e x}^{(\sharp)} \mathbf{x}^{\mathbf{a}^{\prime}} \mathbf{y}^{\mathbf{b}^{\prime}}$ if either (i) $\mathbf{x}^{\mathbf{a}}<_{\text {lex }} \mathbf{x}^{\mathbf{a}^{\prime}}$ or (ii) $\mathbf{x}^{\mathbf{a}}=\mathbf{x}^{\mathbf{a}^{\prime}}$ and $\mathbf{y}^{\mathbf{b}}<^{(\sharp)} \mathbf{y}^{\mathbf{b}^{\prime}}$. Here each of $\mathbf{x}^{\mathbf{a}}$ and $\mathbf{x}^{\mathbf{a}^{\prime}}$ is a monomial belonging to $R$ and each of $\mathbf{y}^{\mathbf{b}}$ and $\mathbf{y}^{\mathbf{b}^{\prime}}$ is a monomial belonging to $K\left[\left\{y_{u}\right\}_{u \in G(I)}\right]$.

We now state the reason why we are interested in prestable ideals. We refer the reader to, e.g., [3] for fundamental materials on Gröbner bases. 
Since a prestable set satisfies the $\ell$-exchange property [7] for all monomial orders $<^{(\sharp)}$ on $K\left[\left\{y_{u}\right\}_{u \in G(I)}\right]$, Theorem 1.2 below is a special case of [7, Theorem 5.1]. However, for the sake of completeness, a proof of Theorem 1.2 will be given.

THEOREM 1.2. Work with the same notation as above. Suppose that $I \subset R$ is a prestable ideal and $\mathscr{G}$ is a Gröbner basis of $J_{\mathscr{R}(I)} \cap K\left[\left\{y_{u}\right\}_{u \in G(I)}\right]$ with respect to $<^{(\sharp)}$. Then

$$
\mathscr{G} \cup\left\{x_{i} y_{u}-x_{j} y_{v} ; u, v \in G(I), x_{i} u=x_{j} v\right\}
$$

is a Gröbner basis of $J_{\mathscr{R}(I)}$ with respect to $<_{\text {lex }}^{(\sharp)}$.

Proof. It is known, e.g., [12] that the reduced Gröbner basis of the toric ideal $J_{\mathscr{R}(I)}$ consists of binomials. Let $f=x_{i_{1}} \cdots x_{i_{p}} y_{u_{1}} \cdots y_{u_{q}}-x_{j_{1}} \cdots x_{j_{p}} y_{v_{1}}$ $\cdots y_{v_{q}}$ be an irreducible binomial belonging to $J_{\mathscr{R}(I)}$ with $p \geq 1$, where $i_{1} \leq$ $i_{2} \leq \cdots$ and $j_{1} \leq j_{2} \leq \cdots$ with $i_{1}<j_{1}$. Thus the initial monomial of $f$ is $i n_{<_{l e x}^{(t)}}(f)=x_{i_{1}} \cdots x_{i_{p}} y_{u_{1}} \cdots y_{u_{q}}$. Write $u_{1} u_{2} \cdots u_{q}=x_{1}^{a_{1}} x_{2}^{a_{2}} \cdots x_{n}^{a_{n}}$ and $v_{1} v_{2} \cdots v_{q}=x_{1}^{b_{1}} x_{2}^{b_{2}} \cdots x_{n}^{b_{n}}$. Then $a_{1}=b_{1}, \ldots, a_{i_{1}-1}=b_{i_{1}-1}$ and $a_{i_{1}}<b_{i_{1}}$. Since $I$ is prestable, it follows that there exist $i$ and $j$ with $1 \leq i \leq q$ and $i_{1}<j \leq n$ such that $x_{i_{1}}\left(u_{i} / x_{j}\right) \in G(I)$. Let $w=x_{i_{1}}\left(u_{i} / x_{j}\right)$. Then $g=x_{i_{1}} y_{u_{i}}-x_{j} y_{w} \in J_{\mathscr{R}(I)}$. Since $x_{j} y_{w}<_{l e x}^{(\sharp)} x_{i_{1}} y_{u_{i}}$, the initial monomial of $g$ is $i n_{<_{l e x}^{(\sharp)}}(g)=x_{i_{1}} y_{u_{i}}$. Thus $i n_{<_{l e x}^{(\sharp)}}(g)$ divides $i n_{<_{l e x}^{(\sharp)}}(f)$.

The elimination property of the lexicographic order together with Theorem 1.2 guarantees that

Corollary 1.3. Suppose that $I \subset R$ is a prestable ideal and $\mathscr{G}$ is a Gröbner basis of $J_{\mathscr{R}(I)} \cap K\left[\left\{y_{u}\right\}_{u \in G(I)}\right]$ with respect to $<^{(\sharp)}$. Then for each $1 \leq \ell \leq n$

$$
\mathscr{G} \cup\left\{x_{i} y_{u}-x_{j} y_{v} ; u, v \in G(I), \ell \leq i, j \leq n, x_{i} u=x_{j} v\right\}
$$

is a Gröbner basis of

$$
J_{\mathscr{R}(I)} \cap K\left[x_{\ell}, x_{\ell+1}, \ldots, x_{n},\left\{y_{u}\right\}_{u \in G(I)}\right]
$$

with respect to $<_{\text {lex }}^{(\sharp)}$ on $K\left[x_{\ell}, x_{\ell+1}, \ldots, x_{n},\left\{y_{u}\right\}_{u \in G(I)}\right]$.

COROLlary 1.4. Let $I \subset R$ be a prestable ideal and suppose that $J_{\mathscr{R}(I)} \cap$ $K\left[\left\{y_{u}\right\}_{u \in G(I)}\right]$ possesses a quadratic Gröbner basis. Then the toric ideal $J_{\mathscr{R}(I)}$ has a quadratic Gröbner basis. Thus in particular the Rees algebra $\mathscr{R}(I)$ is Koszul. 
A monomial ideal $I$ is called strongly stable if, for all $u \in G(I)$ and for all $x_{j}$ that divies $u$, one has $x_{i}\left(u / x_{j}\right) \in G(I)$ for all $i<j$. Conca and De Negri [2] discovered an example of a strongly stable ideal $I \subset R$ for which $J_{\mathscr{R}(I)} \cap K\left[\left\{y_{u}\right\}_{u \in G(I)}\right]$ possesses no quadratic Gröbner basis.

If $f \in A$ is a homogeneous polynomial, then its $x$-degree is the degree $\operatorname{deg}_{x}(f)$ of $f$ as a polynomial in the variables $x_{1}, \ldots, x_{n}$ with coefficients in $K\left[\left\{y_{u}\right\}_{u \in G(I)}\right]$. For example, if $f=x_{1}^{2} x_{2} y_{1} y_{2}-x_{3}^{3} y_{3} y_{4}$, then its $x$-degree is $\operatorname{deg}_{x}(f)=3$. We say that $J_{\mathscr{R}(I)}$ satisfies the $x$-condition [8] if there exists a Gröbner basis $\mathscr{G}$ of $J_{\mathscr{R}(I)}$ such that $\operatorname{deg}_{x}(g) \leq 1$ for each $g \in \mathscr{G}$.

COROLlaRY 1.5. Let I $\subset R$ be a prestable ideal. Then all powers of I have linear resolutions.

Proof. If $I \subset R$ is a prestable ideal, then the toric ideal $J_{\mathscr{R}(I)}$ satisfies the $x$-condition. Thus [8, Corollary 1.2] says that all powers of $I$ have linear resolutions.

\section{Prestable ideals arising from pure posets}

In the present section we are interested in finding a reasonable class of prestable ideals $I \subset R$ for which $J_{\mathscr{R}(I)} \cap K\left[\left\{y_{u}\right\}_{u \in G(I)}\right]$ has a quadratic Gröbner basis.

Let $\Omega=\Omega_{1} \cup \Omega_{2} \cup \cdots \cup \Omega_{d}$ be a finite pure poset [11, p. 99] of rank $d-1$, where each $\Omega_{i}$ is the set of rank $i-1$ elements of $\Omega$. Recall that a poset of rank $d-1$ is called pure if the length of every maximal chain is equal to $d-1$. Let $\Omega_{i}=\left\{x_{1}^{(i)}, x_{2}^{(i)}, \cdots, x_{p_{i}}^{(i)}\right\}$, and let $R=K\left[\left\{x_{j}^{(i)}\right\}_{\substack{1 \leq i \leq d \\ 1 \leq j \leq p_{i}}}\right]$ denote the polynomial ring over a field $K$ with each $\operatorname{deg} x_{j}^{(i)}=1$. We will associate each maximal chain

$$
C: x_{q_{1}}^{(1)}<x_{q_{2}}^{(2)}<\cdots<x_{q_{d}}^{(d)}
$$

of $\Omega$ with the squarefree monomial

$$
x_{C}=x_{q_{1}}^{(1)} x_{q_{2}}^{(2)} \cdots x_{q_{d}}^{(d)}
$$

of $R$ of degree $d$. Let $\mathscr{M}(\Omega)$ denote the set of maximal chains of $\Omega$.

THEOREM 2.1. Work with the same notation as above. Suppose that $\Omega$ satisfies the condition that if $x_{j}^{(i)}>x_{k}^{(i-1)}$, then $x_{j^{\prime}}^{(i)}>x_{k}^{(i-1)}$ for all $j^{\prime}$ with $j^{\prime}<j$ and $x_{j}^{(i)}>x_{k^{\prime}}^{(i-1)}$ for all $k^{\prime}$ with $k<k^{\prime}$. Then $\left\{x_{C} ; C \in M(\Omega)\right\}$ is a prestable set.

Proof. We work with the ordering

$$
x_{1}^{(d)}, x_{2}^{(d)}, \cdots, x_{p_{d}}^{(d)}, x_{1}^{(d-1)}, \cdots, x_{p_{d-1}}^{(d-1)}, x_{1}^{(d-2)}, \cdots, x_{p_{2}}^{(2)}, x_{1}^{(1)}, \cdots, x_{p_{1}}^{(1)} .
$$


Let $\prod_{\lambda=1}^{N} x_{C_{p_{\lambda}}}=\prod_{i, j} x_{j}^{(i)^{a_{j}^{(i)}}}$ and $\prod_{\mu=1}^{N} x_{C_{q_{\mu}}}=\prod_{i, j} x_{j}^{(i)_{j}^{(i)}}$. Suppose that $a_{j_{0}}^{\left(i_{0}\right)}<b_{j_{0}}^{\left(i_{0}\right)}$ and $a_{j}^{(i)}=b_{j}^{(i)}$ for all $i$ and $j$ with either (i) $i>i_{0}$ or (ii) $i=i_{0}$ and $j<j_{0}$. Let $A_{j}=\left\{p_{\lambda} ; x_{j}^{\left(i_{0}\right)} \in C_{p_{\lambda}}\right\}$ and $B_{j}=\left\{q_{\mu} ; x_{j}^{\left(i_{0}\right)} \in C_{q_{\mu}}\right\}$. Then $\sum_{j=1}^{j_{0}}\left|A_{j}\right|<\sum_{j=1}^{j_{0}}\left|B_{j}\right|$, where $\left|A_{j}\right|$ stands for the cardinality of the finite set $A_{j}$. It then follows that there exist $C_{p_{\lambda}}$ and $C_{q_{\mu}}$ such that $C_{p_{\lambda}} \cap \Omega_{i_{0}+1}=$ $C_{q_{\mu}} \cap \Omega_{i_{0}+1}, C_{p_{\lambda}} \cap \Omega_{i_{0}} \subset\left\{x_{j_{0}+1}^{\left(i_{0}\right)}, \ldots, x_{p_{i_{0}}}^{\left(i_{0}\right)}\right\}$ and $C_{q_{\mu}} \cap \Omega_{i_{0}} \subset\left\{x_{1}^{\left(i_{0}\right)}, \ldots, x_{j_{0}}^{\left(i_{0}\right)}\right\}$. Let $C_{p_{\lambda}} \cap \Omega_{i_{0}+1}=\left\{x_{r}^{\left(i_{0}+1\right)}\right\}, C_{p_{\lambda}} \cap \Omega_{i_{0}}=\left\{x_{j^{\prime}}^{\left(i_{0}\right)}\right\}$ and $C_{q_{\mu}} \cap \Omega_{i_{0}}=\left\{x_{j^{\prime \prime}}^{\left(i_{0}\right)}\right\}$. Then $j^{\prime \prime} \leq j_{0}<j^{\prime}$. Since $x_{j^{\prime \prime}}^{\left(i_{0}\right)}<x_{r}^{\left(i_{0}+1\right)}$ in $\Omega$, one has $x_{j_{0}}^{\left(i_{0}\right)}<x_{r}^{\left(i_{0}+1\right)}$. Let $C_{p_{\lambda}} \cap \Omega_{i_{0}-1}=\left\{x_{k}^{\left(i_{0}-1\right)}\right\}$. Since $x_{k}^{\left(i_{0}-1\right)}<x_{j^{\prime}}^{\left(i_{0}\right)}$ in $\Omega$, one has $x_{k}^{\left(i_{0}-1\right)}<x_{j_{0}}^{\left(i_{0}\right)}$. Hence $x_{k}^{\left(i_{0}-1\right)}<x_{j_{0}}^{\left(i_{0}\right)}<x_{r}^{\left(i_{0}+1\right)}$. Thus $\left(C_{p_{\lambda}} \backslash\left\{x_{j^{\prime}}^{\left(i_{0}\right)}\right\}\right) \cup\left\{x_{j_{0}}^{\left(i_{0}\right)}\right\} \in \mathcal{M}(\Omega)$, as required.

Corollary 2.2. Suppose that $\Omega$ satisfies the same condition as in Theorem 2.1 and let $I \subset R$ denote the prestable ideal with $G(I)=\left\{x_{C} ; C \in\right.$ $\mathcal{M}(\Omega)\}$. Then all powers of I have linear resolutions. Moreover, the toric ideal $J_{\mathscr{R}(I)}$ of the Rees algebra $\mathscr{R}(I)$ possesses a squarefree and quadratic Gröbner basis. (A Gröbner basis is called squarefree if the initial monomial of each polynomial belonging to the Gröbner basis is squarefree.)

Proof. By virtue of Theorem 2.1 together with Corollary 1.5 it follows that all powers of $I$ have linear resolutions. By using Corollary 1.4, in order to show that $J_{\mathscr{R}(I)}$ has a squarefree and quadratic Gröbner basis, our work is to prove that $J_{\mathscr{R}(I)} \cap K\left[\left\{y_{u}\right\}_{u \in G(I)}\right]$ has a squarefree and quadratic Gröbner basis.

Let $<_{\text {rev }}$ denote the reverse lexicographic order on $R=K\left[\left\{x_{j}^{(i)}\right\}_{\substack{1 \leq i \leq d \\ 1 \leq j \leq p_{i}}}\right]$ induced by the same ordering as in the proof of Theorem 2.1. Thus $u_{x_{1}^{(d)}}$ is the biggest variable and $u_{x_{p_{1}}^{(1)}}$ is the smallest variable with respect to $<_{\text {rev }}$. We then introduce the reverse lexicographic order $<^{(\sharp)}$ on $K\left[\left\{y_{u}\right\}_{u \in G(I)}\right]$ such that $y_{u}<^{(\sharp)} y_{v}$ if $u<_{\text {rev }} v$.

Let $C_{\alpha}, C_{\beta} \in \mathcal{M}(\Omega)$. We say that $\left(C_{\alpha}, C_{\beta}\right)$ is a noncrossing pair if $C_{\alpha} \cap$ $\Omega_{i}=\left\{x_{j_{i}(\alpha)}^{(i)}\right\}$ and $C_{\beta} \cap \Omega_{i}=\left\{x_{j_{i}(\beta)}^{(i)}\right\}$, then $j_{i}(\alpha) \leq j_{i}(\beta)$ for all $1 \leq i \leq d$.

Let $C_{\lambda}, C_{\mu} \in \mathcal{M}(\Omega)$ for which $\left(C_{\lambda}, C_{\mu}\right)$ is a crossing pair, i.e., there is $1 \leq i_{0}<d$ such that if $C_{\lambda} \cap \Omega_{i_{0}+1}=\left\{x_{j}^{\left(i_{0}+1\right)}\right\}, C_{\mu} \cap \Omega_{i_{0}+1}=\left\{x_{j^{\prime}}^{\left(i_{0}+1\right)}\right\}$, $C_{\lambda} \cap \Omega_{i_{0}}=\left\{x_{k}^{\left(i_{0}\right)}\right\}$ and $C_{\mu} \cap \Omega_{i_{0}}=\left\{x_{k^{\prime}}^{\left(i_{0}\right)}\right\}$, then $j^{\prime}<j$ and $k<k^{\prime}$. Since $\Omega$ satisfies the same condition as in Theorem 2.1, it follows that $x_{k}^{\left(i_{0}\right)}<x_{j^{\prime}}^{\left(i_{0}+1\right)}$ and $x_{k^{\prime}}^{\left(i_{0}\right)}<x_{j}^{\left(i_{0}+1\right)}$. Let $C_{\lambda^{\prime}}=\left(C_{\lambda} \cap\left(\cup_{i=i_{0}+1}^{d} \Omega_{i}\right)\right) \cup\left(C_{\mu} \cap\left(\cup_{i=1}^{i_{0}} \Omega_{i}\right)\right)$ and $C_{\mu^{\prime}}=\left(C_{\mu} \cap\left(\cup_{i=i_{0}+1}^{d} \Omega_{i}\right)\right) \cup\left(C_{\lambda} \cap\left(\cup_{i=1}^{i_{0}} \Omega_{i}\right)\right)$. Then $C_{\lambda^{\prime}}, C_{\mu^{\prime}} \in \mathcal{M}(\Omega)$ and $x_{C_{\lambda}} x_{C_{\mu}}=x_{C_{\lambda^{\prime}}} x_{C_{\mu^{\prime}}}$. Repeated applications of such the technique guarantees the existence of $C_{\alpha}, C_{\beta} \in \mathscr{M}(\Omega)$ such that $\left(C_{\alpha}, C_{\beta}\right)$ is a noncrossing pair 
with $x_{C_{\lambda}} x_{C_{\mu}}=x_{C_{\alpha}} x_{C_{\beta}}$. The existence of a noncrossing pair $\left(C_{\alpha}, C_{\beta}\right)$ with $x_{C_{\lambda}} x_{C_{\mu}}=x_{C_{\alpha}} x_{C_{\beta}}$ is unique and $u_{x_{C_{\alpha}}} u_{x_{C_{\beta}}}{ }^{(\sharp)} u_{x_{C_{\lambda}}} u_{x_{C_{\mu}}}$.

Hence by using the well-known technique, e.g., [10, Lemma 0.1], it turns out that the set of all binomials $u_{x_{C_{\lambda}}} u_{x_{C_{\mu}}}-u_{x_{C_{\alpha}}} u_{x_{C_{\beta}}}$, where $\left(C_{\alpha}, C_{\beta}\right)$ is a noncrossing pair, $\left(C_{\lambda}, C_{\mu}\right)$ is a crossing pair and $x_{C_{\lambda}} x_{C_{\mu}}=x_{C_{\alpha}} x_{C_{\beta}}$ is a Gröbner basis of $J_{\mathscr{R}(I)} \cap K\left[\left\{y_{u}\right\}_{u \in G(I)}\right]$ with respect to $<^{(\sharp)}$.

Example 2.3. Let $R=K\left[\left\{x_{j}^{(i)}\right\}_{\substack{1 \leq i \leq n \\ 1<i<m}}\right]$, where $n \leq m$, denote the polynomial ring in $\mathrm{nm}$ variables and $\mathcal{N}$ the set of all (squarefree) monomials $x_{j_{1}}^{(1)} x_{j_{2}}^{(2)} \ldots x_{j_{n}}^{(n)}$ with $1 \leq j_{1}<j_{2}<\ldots<j_{n} \leq m$. Let $I \subset R$ be the ideal with $G(I)=\mathscr{N}$. Then all powers of $I$ have linear resolutions and the toric ideal $J_{\mathscr{R}(I)}$ of the Rees algebra $\mathscr{R}(I)$ possesses a squarefree and quadratic Gröbner basis. It follows from Corollary 1.3 that, for each $1 \leq \ell \leq n$, the ideal $J_{\mathscr{R}(I)} \cap K\left[\left\{x_{j}^{(i)}\right\}_{\substack{\ell \leq i \leq n \\ 1 \leq i \leq m}},\left\{y_{u}\right\}_{u \in G(I)}\right]$ (as well as the ideal $\left.J_{\mathscr{R}(I)} \cap K\left[\left\{x_{j}^{(i)}\right\}_{\substack{1 \leq i \leq \ell \\ 1 \leq j \leq m}},\left\{y_{u}\right\}_{u \in G(I)}\right]\right)$ possesses a squarefree and quadratic Gröbner basis.

\section{Computations of Sagbi bases}

Let $K[\mathbf{t}]=K\left[t_{1}, \ldots, t_{d}\right]$ denote the polynomial ring in $d$ variables over a field $K$. Given a finite set $\mathscr{F}=\left\{f_{1}, \ldots, f_{n}\right\} \subset K[\mathbf{t}]$, we write $K[\mathscr{F}]=$ $K\left[f_{1}, \ldots, f_{n}\right]$ for the subalgebra of $K[\mathbf{t}]$ generated by $f_{1}, \ldots, f_{n}$. The initial algebra of $K[\mathscr{F}]$ with respect to a monomial order $<$ on $K[\mathbf{t}]$ is the subalgebra

$$
\operatorname{in}_{<}(K[\mathscr{F}])=K\left[\left\{i n_{<}(f) ; f \in K[\mathscr{F}]\right\}\right]
$$

of $K[\mathbf{t}]$. A subset $\mathscr{S}$ of $K[\mathscr{F}]$ is said to be a Sagbi basis of $K[\mathscr{F}]$ with respect to $<$ if $i n_{<}(K[\mathscr{F}])$ is generated by $\left\{i n_{<}(s) ; s \in \mathscr{S}\right\}$. A Sagbi basis always exists. However, a finite Sagbi basis does not necessarily exist.

Let $R=K\left[x_{1}, \ldots, x_{n}\right]$ denote the polynomial ring in $n$ variables over $K$ with each $\operatorname{deg} x_{i}=1$ and $I_{\mathscr{F}}(\subset R)$ the defining ideal of $K[\mathscr{F}]$. Thus $I_{\mathscr{F}}$ is the kernel of the surjective ring homomorphism from $R$ to $K[\mathscr{F}]$ defined by setting $x_{i} \mapsto f_{i}$.

Given a generic weight vector $w \in \mathbf{R}_{>0}^{d}$ on $K[\mathbf{t}]$, we introduce the new weight vector $\tilde{w}=\left(w \cdot \mathbf{a}_{1}, \ldots, w \cdot \mathbf{a}_{n}\right) \in \mathbf{R}_{\geq 0}^{n}$ on $R$, where $i n_{w}\left(f_{i}\right)=\mathbf{t}^{\mathbf{a}_{i}}=$ $t_{1}^{a_{i}^{(1)}} \cdots t_{d}^{a_{i}^{(d)}}$ with $\mathbf{a}_{i}=\left(a_{i}^{(1)}, \ldots, a_{i}^{(d)}\right)$. The initial ideal $i n_{\tilde{w}}\left(I_{\mathscr{F}}\right)$ of $I_{\mathscr{F}}$ with respect to $\tilde{w}$ may not be a monomial ideal. Let $J_{i n_{w}(\mathscr{F})}(\subset R)$ denote the toric ideal of the semigroup ring $K\left[i n_{w}\left(f_{1}\right), \ldots, i n_{w}\left(f_{n}\right)\right]$. It is known [12, Lemma 11.3] that $i n_{\tilde{w}}\left(I_{\mathscr{F}}\right) \subset J_{i n_{w}(\mathscr{F})}$. Moreover, [12, Theorem 11.4] says that

Lemma 3.1. A subset $\mathscr{F} \subset K[\mathscr{F}]$ is a Sagbi basis of $K[\mathscr{F}]$ with respect to a weight vector $w$ if and only if $J_{i n_{w}(\mathscr{F})} \subset i n_{\tilde{w}}\left(I_{\mathscr{F}}\right)$. 
Fix integers $1 \leq \ell<n \leq m$. Let $X=\left(x_{j}^{(i)}\right)_{\substack{1 \leq i \leq n \\ 1 \leq j \leq m}}$ be the $n \times m$ matrix of variables and $K[X]=K\left[\left\{x_{j}^{(i)}\right\}_{\substack{1 \leq i \leq n \\ 1 \leq j \leq m}}\right.$ the polynomial ring over $K$. The notation $\left[j_{1}, j_{2}, \ldots, j_{n}\right], 1 \leq j_{1}<j_{2}<\cdots<j_{n} \leq m$, stands for the $n \times n$ submatrix

$$
\left(\begin{array}{ccc}
x_{j_{1}}^{(1)} x_{j_{2}}^{(1)} & \ldots & x_{j_{n}}^{(1)} \\
x_{j_{1}}^{(2)} x_{j_{2}}^{(2)} & \ldots & x_{j_{n}}^{(2)} \\
\vdots & & \vdots \\
x_{j_{1}}^{(n)} x_{j_{2}}^{(n)} & \ldots & x_{j_{n}}^{(n)}
\end{array}\right)
$$

of $X$. Let $X_{\ell}=\left\{x_{j}^{(i)} ; 1 \leq i \leq \ell, 1 \leq j \leq m\right\}$ and

$$
\Gamma(X)=\left\{\operatorname{det}\left(\left[j_{1}, j_{2}, \ldots, j_{n}\right]\right) ; 1 \leq j_{1}<j_{2}<\cdots<j_{n} \leq m\right\} .
$$

In [5] the authors discuss the subalgebra $R_{\ell}(X)=K\left[\Gamma(X) \cup X_{\ell}\right]$ of $K[X]$. If $\ell=1$, then a Sagbi basis of $R_{1}(X)$ is given in [9, Proposition 2.1] (when $n=2$ ) and [6] (when $n \geq 2$ ). Using Example 2.3 we determine a Sagbi basis of $R_{\ell}(X)$ for all $\ell \geq 1$.

Recall that a diagonal order on $K[X]$ is a monomial order $<$ on $K[X]$ such that

$$
i n_{<}\left(\operatorname{det}\left(\left[j_{1}, j_{2}, \ldots, j_{n}\right]\right)\right)=x_{j_{1}}^{(1)} x_{j_{2}}^{(2)} \cdots x_{j_{n}}^{(n)}
$$

for all $\operatorname{det}\left(\left[j_{1}, j_{2}, \ldots, j_{n}\right]\right) \in \Gamma(X)$. We work with the diagonal order $<_{\text {diag }}$ on $K[X]$ defined by the weight vector $w=\left(i^{j}\right)_{\substack{1 \leq i \leq n \\ 1 \leq j \leq m}}^{\substack{c \\ \text {. }}}$

THeOREM 3.2. The finite set $\Gamma(X) \cup X_{\ell}$ is a Sagbi basis of $R_{\ell}(X)$ with respect to the weight vector $w=\left(i^{j}\right)_{\substack{1 \leq i \leq n \\ 1 \leq i \leq m}}$. Moreover, the toric ideal $J_{i n_{w}\left(\Gamma(X) \cup X_{\ell}\right)}$ possesses a squarefree and quadratic initial ideal. In particular the initial algebra in $_{w}\left(R_{\ell}(X)\right)$ is normal and Koszul.

Proof. Example 2.3 says that the toric ideal $J_{i n_{w}(\Gamma(X))}$ possesses a squarefree quadratic Gröbner basis $\mathscr{G}$ and that

$$
\mathscr{G} \cup\left\{x_{j}^{(i)} y_{u}-x_{j^{\prime}}^{(i)} y_{v} ; u, v \in \mathcal{N}, x_{j}^{(i)} u=x_{j^{\prime}}^{(i)} v, 1 \leq i \leq \ell\right\}
$$

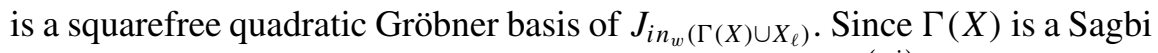
basis of $K[\Gamma(X)]$ with respect to the weight vector $w=\left(i^{j}\right)_{\substack{1 \leq i \leq n \\ 1 \leq j \leq m}}([12$, Theorem 11.8]), it follows that $\mathscr{G} \subset i n_{\tilde{w}}\left(I_{\Gamma(X)}\right) \subset i n_{\tilde{w}}\left(I_{\Gamma(X) \cup X_{\ell}}\right)$. By virtue of Lemma 3.1, our work is to show that each binomial $x_{j}^{(i)} y_{u}-x_{j^{\prime}}^{(i)} y_{v} \in$ $J_{i n_{w}\left(\Gamma(X) \cup X_{\ell}\right)}$ belongs to $i n_{\tilde{w}}\left(I_{\Gamma(X) \cup X_{\ell}}\right)$.

Let $x_{j}^{(i)} y_{u}-x_{j^{\prime}}^{(i)} y_{v} \in J_{i n_{w}\left(\Gamma(X) \cup X_{\ell}\right)}$. Then there exist

$$
1 \leq k_{1}<\cdots<k_{i-1}<j<j^{\prime}<k_{i+1}<\cdots<k_{n} \leq m
$$


such that $u($ resp. $v)$ is the main diagonal monomial of $\operatorname{det}(U)(\operatorname{resp} \cdot \operatorname{det}(V))$, where $U=\left[k_{1}, \ldots, k_{i-1}, j^{\prime}, k_{i+1}, \ldots, k_{n}\right]$ (resp. $V=\left[k_{1}, \ldots, k_{i-1}, j, k_{i+1}\right.$, $\left.\left.\ldots, k_{n}\right]\right)$. Now, we introduce the $(n+1) \times(n+1)$ matrix

$$
M=\left(\begin{array}{cccccc}
x_{k_{1}}^{(1)} & \ldots & x_{j}^{(1)} & x_{j^{\prime}}^{(1)} & \ldots & x_{k_{n}}^{(1)} \\
\vdots & & \vdots & \vdots & & \vdots \\
x_{k_{1}}^{(i)} & \ldots & x_{j}^{(i)} & x_{j^{\prime}}^{(i)} & \ldots & x_{k_{n}}^{(i)} \\
x_{k_{1}}^{(i)} & \ldots & x_{j}^{(i)} & x_{j^{\prime}}^{(i)} & \ldots & x_{k_{n}}^{(i)} \\
\vdots & & \vdots & \vdots & & \vdots \\
x_{k_{1}}^{(n)} & \ldots & x_{j}^{(n)} & x_{j^{\prime}}^{(n)} & \ldots & x_{k_{n}}^{(n)}
\end{array}\right)
$$

with $\operatorname{det}(M)=0$. Since

$$
\begin{aligned}
\operatorname{det}(M)=\sum_{p=1}^{i-1} & (-1)^{p} x_{k_{p}}^{(i)} \operatorname{det}\left(k_{1}, \ldots, k_{p-1}, k_{p+1}, \ldots, j, j^{\prime}, \ldots, k_{n}\right) \\
& +(-1)^{i} x_{j}^{(i)} \operatorname{det}(U)+(-1)^{i+1} x_{j^{\prime}}^{(i)} \operatorname{det}(V) \\
& -\sum_{p=i+1}^{n}(-1)^{p} x_{k_{p}}^{(i)} \operatorname{det}\left(k_{1}, \ldots, j, j^{\prime}, \ldots, k_{p-1}, k_{p+1}, \ldots, k_{n}\right),
\end{aligned}
$$

the polynomial

$$
f=(-1)^{i}\left(x_{j}^{(i)} y_{u}-x_{j^{\prime}}^{(i)} y_{v}\right)+\sum_{p=1}^{i-1}(-1)^{p} x_{k_{p}}^{(i)} y_{u_{p}}-\sum_{p=i+1}^{n}(-1)^{p} x_{k_{p}}^{(i)} y_{u_{p}},
$$

where each of the $u_{p}$ 's is equal to either $\operatorname{det}\left[k_{1}, \ldots, k_{p-1}, k_{p+1}, \ldots, j, j^{\prime}, \ldots\right.$, $\left.k_{n}\right]$ or $\operatorname{det}\left[k_{1}, \ldots, j, j^{\prime}, \ldots, k_{p-1}, k_{p+1}, \ldots, k_{n}\right]$, belongs to the defining ideal $I_{\Gamma(X) \cup X_{\ell}}$. Since the main diagonal monomial of $\operatorname{det}(M)$ is $i n_{w}\left(x_{j}^{(i)} \operatorname{det}(U)\right)=$ $i n_{w}\left(x_{j^{\prime}}^{(i)} \operatorname{det}(V)\right)$, it follows that $i n_{\tilde{w}}(f)=x_{j}^{(i)} y_{u}-x_{j^{\prime}}^{(i)} y_{v}$, as desired.

\section{REFERENCES}

1. Blum, S., Subalgebras of bigraded Koszul algebras, J. Algebra 242 (2001), 795-809.

2. Conca, A., and De Negri, E., M-sequences, graph ideals, and ladder ideals of linear type, J. Algebra 211 (1999), 599-624.

3. Cox, D., Little, J., and O'Shea, D., Ideals, Varieties and Algorithms, Springer-Verlag, Berlin, Heidelberg, New York, 1992.

4. Eliahou, S., and Kervaire, M., Minimal resolutions of some monomial ideals, J. Algebra 129 (1990), 1-25. 
5. Goto, S., Hayasaka, F., Kurano, K., and Nakamura, Y., Algebras generated by the entries of a certain submatrix and the maximal minors, preprint, 2003.

6. Hayasaka, F., Rees algebras associated to modules, in Proceedings of the 15th seminar on commutative algebra in Japan (Japanese) (Nara, 2003), in press.

7. Herzog, J., Hibi, T., and Vladoiu, M., Ideals of fiber type and polymatroids, preprint, 2003.

8. Herzog, J., Hibi, T., and Zheng, X., Monomial ideals whose powers have a linear resolution, preprint, 2003.

9. Herzog, J., Tang, Z., and Zarzuela, S., Symmetric and Rees algebras of Koszul cycles and their Gröbner bases, Manuscripta Math. 112 (2003), no. 4, 489-509.

10. Ohsugi, H., and Hibi, T., Normalized volumes of configurations related with root systems and complete bipartite graphs, Discrete Math. 268 (2003), 217-242.

11. Stanley, R., Enumerative Combinatorics, Volume I, Wadsworth \& Brooks/Cole, Monterey, CA, 1986.

12. Sturmfels, B., Gröbner Bases and Convex Polytopes, Amer. Math. Soc., Providence, RI, 1995.

DEPARTMENT OF MATHEMATICS

FACULTY OF SCIENCE

RIKKYO UNIVERSITY

TOSHIMA, TOKYO 171-8501

JAPAN

E-mail: ohsugi@rkmath.rikkyo.ac.jp
DEPARTMENT OF PURE AND APPLIED MATHEMATICS GRADUATE SCHOOL OF INFORMATION SCIENCE AND TECHNOLOGY

OSAKA UNIVERSITY

TOYONAKA, OSAKA 560-0043

JAPAN

E-mail: hibi@math.sci.osaka-u.ac.jp 